\title{
Inequalities of Green's functions and positive solutions to nonlocal boundary value problems
}

Shijie Fan ${ }^{1}$, Pengxu Wen ${ }^{1}$ and Guowei Zhang ${ }^{1 *}$

\section{"Correspondence:}

gwzhang@mail.neu.edu.cn; gwzhangneum@sina.com

'Department of Mathematics, Northeastern University, Shenyang, China

\begin{abstract}
In this paper, we discuss the positive solutions of beam equations with the nonlinearities including the slope and bending moment under nonlocal boundary conditions involving Stieltjes integrals. We pose some inequality conditions on nonlinearities and the spectral radius conditions on associated linear operators. These conditions mean that the nonlinearities have superlinear or sublinear growth. The existence of positive solutions is obtained by fixed point index on cones in $C^{2}[0,1]$, and some examples are given for beam equations subject to mixed integral and multi-point boundary conditions with sign-changing coefficients.
\end{abstract}

MSC: Primary 34B18; 34B10; secondary 34B15

Keywords: Positive solution; Fixed point index; Cone; Spectral radius

\section{Introduction and preliminaries}

In this paper, we discuss the existence of positive solutions to fourth-order boundary value problems (BVPs):

$$
\left\{\begin{array}{l}
u^{(4)}(t)=f\left(t, u(t), u^{\prime}(t), u^{\prime \prime}(t)\right), \quad t \in[0,1], \\
u(0)=\beta_{1}[u], \quad u^{\prime}(1)=\beta_{2}[u], \\
u^{\prime \prime}(0)+\beta_{3}[u]=0, \quad u^{\prime \prime}(1)+\beta_{4}[u]=0,
\end{array}\right.
$$

and

$$
\left\{\begin{array}{l}
-u^{(4)}(t)=g\left(t, u(t), u^{\prime}(t), u^{\prime \prime}(t)\right), \quad t \in[0,1], \\
u(0)=\alpha_{1}[u], \quad u^{\prime}(0)=\alpha_{2}[u], \quad u^{\prime \prime}(0)=\alpha_{3}[u], \quad u^{\prime \prime}(1)=\alpha_{4}[u],
\end{array}\right.
$$

where $\beta_{i}[u], \alpha_{i}[u](i=1,2,3,4)$ are given by

$$
\beta_{i}[u]=\int_{0}^{1} u(t) d B_{i}(t) \quad \text { and } \quad \alpha_{i}[u]=\int_{0}^{1} u(t) d A_{i}(t)
$$

(c) The Author(s) 2020. This article is licensed under a Creative Commons Attribution 4.0 International License, which permits use, sharing, adaptation, distribution and reproduction in any medium or format, as long as you give appropriate credit to the original author(s) and the source, provide a link to the Creative Commons licence, and indicate if changes were made. The images or other third party material in this article are included in the article's Creative Commons licence, unless indicated otherwise in a credit line to the material. If material is not included in the article's Creative Commons licence and your intended use is not permitted by statutory regulation or exceeds the permitted use, you will need to obtain permission directly from the copyright holder. To view a copy of this licence, visit http://creativecommons.org/licenses/by/4.0/. 
involving Stieltjes integrals with $B_{i}, A_{i}$ of bounded variation. They model the deflection of beam equations with the nonlinearities including the slope $u^{\prime}$ and bending moment $u^{\prime \prime}$. The boundary conditions of Stieltjes integrals imply that the mechanism at the end points depends on the feedback along parts of the beam to control the displacement.

By monotone iteration method, the cantilever beam equation containing the slope term

$$
u^{(4)}(t)=f\left(t, u(t), u^{\prime}(t)\right)
$$

was considered by Alves et al. [1] and Yao [18] separately under the boundary conditions

$$
u(0)=u^{\prime}(0)=0, \quad u^{\prime \prime \prime}(1)=g(u(1)), \quad u^{\prime}(1)=0 \quad \text { or } \quad u^{\prime \prime}(1)=0,
$$

where $g$ is a continuous function, and

$$
u(0)=u^{\prime}(0)=u^{\prime \prime}(1)=u^{\prime \prime \prime}(1)=0 .
$$

Respectively based on fixed point index method and global bifurcation technique, Li [10] and $\mathrm{Ma}$ [12] were devoted to the beam equations involving the bending moment with the hinged ends

$$
\left\{\begin{array}{l}
u^{(4)}(t)=f\left(t, u(t), u^{\prime \prime}(t)\right), \quad t \in(0,1) \\
u(0)=u^{\prime \prime}(0)=u(1)=u^{\prime \prime}(1)=0
\end{array}\right.
$$

Li [11] was concerned with the cantilever beam equation

$$
\left\{\begin{array}{l}
u^{(4)}(t)=f\left(t, u(t), u^{\prime}(t), u^{\prime \prime}(t), u^{\prime \prime \prime}(t)\right), \quad t \in[0,1] \\
u(0)=u^{\prime}(0)=u^{\prime \prime}(1)=u^{\prime \prime \prime}(1)=0
\end{array}\right.
$$

where $f:[0,1] \times \mathbb{R}_{+}^{3} \times \mathbb{R}_{-} \rightarrow \mathbb{R}_{+}$is continuous. The existence of positive solutions is obtained if the superlinear or sublinear growth conditions are satisfied for the nonlinearity. However, the boundary conditions in [1, 10-12, 18] are all local. Webb et al. [17] considered the existence of positive solutions for the beam equation

$$
u^{(4)}(t)=g(t) f(t, u(t)), \quad \text { a.e. } t \in(0,1)
$$

respectively subject to nonlocal boundary conditions such as

$$
\begin{aligned}
& u(0)=0, \quad u(1)=\alpha[u], \quad u^{\prime}(0)=0, \quad u^{\prime}(1)=0, \\
& u(0)=0, \quad u(1)=0, \quad u^{\prime}(0)=0, \quad u^{\prime}(1)+\alpha[u]=0, \\
& u(0)=0, \quad u^{\prime \prime}(0)=0, \quad u(1)=\alpha[u], \quad u^{\prime \prime}(1)=0,
\end{aligned}
$$

and

$$
u(0)=0, \quad u(1)=0, \quad u^{\prime \prime}(0)=0, \quad u^{\prime \prime}(1)+\alpha[u]=0 .
$$


In these conditions $\alpha[u]=\int_{0}^{1} u(s) d A(s)$ is given by Stieltjes integral. Infante and Pietramala [6] studied the existence of positive solutions for cantilever beam equation

$$
\left\{\begin{array}{l}
u^{(4)}(t)=g(t) f(t, u(t)), \quad t \in(0,1) \\
u(0)=u^{\prime}(0)=u^{\prime \prime}(1)=0, \quad u^{\prime \prime \prime}(1)+k_{0}+B(\alpha[u])=0
\end{array}\right.
$$

where $k_{0}$ is a nonnegative constant, $\alpha[u]$ is Stieltjes integral, and $B$ is a nonnegative continuous function. The nonlinearity $f$ in $[6,17]$ is not affected by the slope and bending moment, and the authors used the method of fixed point index on cone. We also refer to some other articles, for instance, $[2,5,7,9,14,19]$.

Recently, the authors in [13] investigated the existence of positive solutions to the following problems:

$$
\left\{\begin{array}{l}
u^{(4)}(t)=f\left(t, u(t), u^{\prime}(t), u^{\prime \prime}(t), u^{\prime \prime \prime}(t)\right), \quad t \in[0,1], \\
u^{\prime}(0)+\beta_{1}[u]=0, \quad u^{\prime \prime}(0)+\beta_{2}[u]=0, \quad u(1)=\beta_{3}[u], \quad u^{\prime \prime \prime}(1)=0,
\end{array}\right.
$$

and

$$
\left\{\begin{array}{l}
-u^{(4)}(t)=g\left(t, u(t), u^{\prime}(t), u^{\prime \prime}(t), u^{\prime \prime \prime}(t)\right), \quad t \in[0,1], \\
u(0)=\alpha_{1}[u], \quad u^{\prime}(0)=\alpha_{2}[u], \quad u^{\prime \prime}(0)=\alpha_{3}[u], \quad u^{\prime \prime \prime}(1)=0,
\end{array}\right.
$$

where $\beta_{i}[u], \alpha_{i}[u](i=1,2,3)$ are linear functionals involving Stieltjes integrals of signed measures, and the nonlinearities $f, g$ satisfy the Nagumo condition, which restricts $f$ and $g$ on $u^{\prime \prime \prime}$ to quadric growth for the superlinear case, as in Li [11].

If the nonlinearities in (1.3) and (1.4) are independent of $u^{\prime \prime \prime}$, the restriction of quadric growth can certainly be rid of. However, the boundary conditions in (1.1) and (1.2) are different from those in (1.3) and (1.4). Especially the third derivatives with respect to $t$ of their Green's functions corresponding to (1.1) and (1.2) may be sign-changing while they are not corresponding to (1.3) and (1.4), which plays an important part when estimating the norms in [13]. When BVPs are converted to integral equations, a general method due to Webb and Infante [16] is applied to use the theory of fixed point index on cones in $C^{2}[0,1]$. Some examples are given for beam equations subject to mixed integral and multipoint boundary conditions with sign-changing coefficients. We also cite [15] in which a different approach is applied to the existence of positive solutions for the problem

$$
\left\{\begin{array}{l}
u^{(4)}(t)=h(t) f\left(t, u(t), u^{\prime}(t), u^{\prime \prime}(t)\right), \quad t \in(0,1), \\
u(0)=u(1)=\beta_{1}[u], \quad u^{\prime \prime}(0)+\beta_{2}[u]=0, \quad u^{\prime \prime}(1)+\beta_{3}[u]=0,
\end{array}\right.
$$

where $f:[0,1] \times \mathbb{R}_{+} \times \mathbb{R} \times \mathbb{R}_{-} \rightarrow \mathbb{R}_{+}$is continuous, $h \in L^{1}(0,1)$, and $\beta_{i}[u]$ is Stieltjes inte$\operatorname{gral}(i=1,2,3)$.

If the nonempty subset $P$ in Banach space $X$ satisfies the following conditions: (i) it is a closed convex set, (ii) $\lambda x \in P$ for any $\lambda>0, x \in P$, and (iii) $\pm x \in P \Leftrightarrow x=0$ (0 stands for the zero element in $X$ ), then $P$ is said to be a cone in $X$. A cone $P$ is called reproducing if $X=P-P$. It is well known that if $P$ is a solid cone, i.e., the interior point set $\stackrel{\circ}{P} \neq \emptyset, P$ is reproducing. Now we state some properties of fixed point index (see $[3,4])$. 
Lemma 1.1 Let $\Omega$ be a bounded open subset of Banach space $X$ with $0 \in \Omega$ and $P$ be a cone in $X$. If $S: P \cap \bar{\Omega} \rightarrow P$ is a completely continuous operator and $\mu S u \neq u$ for $u \in P \cap \partial \Omega$ and $\mu \in[0,1]$, then the fixed point index $i(S, P \cap \Omega, P)=1$.

Lemma 1.2 Let $\Omega$ be a bounded open subset of Banach space $X$ and $P$ be a cone in $X$. If $S: P \cap \bar{\Omega} \rightarrow P$ is a completely continuous operator and there exists $v_{0} \in P \backslash\{0\}$ such that $u-S u \neq v v_{0}$ for $u \in P \cap \partial \Omega$ and $v \geq 0$, then the fixed point index $i(S, P \cap \Omega, P)=0$.

Lemma 1.3 (Krein-Rutman) Let $P$ be a reproducing cone in Banach space $X$ and $L: X \rightarrow$ $X$ be a completely continuous linear operator with $L(P) \subset P$. If the spectral radius $r(L)>0$, then there exists $\varphi \in P \backslash\{0\}$ such that $L \varphi=r(L) \varphi$.

Lemma 1.4 ([8]) Let P be a cone in Banach space $X$ and $L: X \rightarrow X$ be a completely continuous linear operator with $L(P) \subset P$. If there exist $v_{0} \in P \backslash\{0\}$ and $\lambda_{0}>0$ such that $L v_{0} \geq \lambda_{0} v_{0}$ in the sense of partial ordering induced by $P$, then there exist $u_{0} \in P \backslash\{0\}$ and $\lambda_{1} \geq \lambda_{0}$ such that $L u_{0}=\lambda_{1} u_{0}$.

Throughout this paper, denote the Banach space that consists of all second-order continuously differentiable functions on $[0,1]$ by $X=C^{2}[0,1]$ and the norm by $\|u\|_{C^{2}}=$ $\max \left\{\|u\|_{C},\left\|u^{\prime}\right\|_{C},\left\|u^{\prime \prime}\right\|_{C}\right\}$.

\section{Inequalities of Green's function and positive solutions for (1.1)}

For BVP (1.1) we make the assumption:

$\left(C_{1}\right) f:[0,1] \times \mathbb{R}_{+}^{2} \times \mathbb{R}_{-} \rightarrow \mathbb{R}_{+}$is continuous, here $\mathbb{R}_{+}=[0, \infty)$ and $\mathbb{R}_{-}=(-\infty, 0]$.

Similar to Webb and Infante [16], BVP (1.1) can be converted to integral equation in $C^{2}[0,1]$

$$
u(t)=(S u)(t)=: \int_{0}^{1} k_{S}(t, s) f\left(s, u(s), u^{\prime}(s), u^{\prime \prime}(s)\right) d s,
$$

where

$$
k_{S}(t, s)=\left\langle(I-[B])^{-1} \mathcal{K}(s), \gamma(t)\right\rangle+k_{0}(t, s)=\sum_{i=1}^{4} \kappa_{i}(s) \gamma_{i}(t)+k_{0}(t, s),
$$

$\left\langle(I-[B])^{-1} \mathcal{K}(s), \gamma(t)\right\rangle$ is the inner product in $\mathbb{R}^{4}$,

$$
\begin{array}{ll}
\mathcal{K}_{i}(s)=\int_{0}^{1} k_{0}(t, s) d B_{i}(t) & (i=1,2,3,4), \\
\gamma_{1}(t) & =1, \quad \gamma_{2}(t)=t, \quad \gamma_{3}(t)=\frac{1}{6} t\left(t^{2}-3 t+3\right), \quad \gamma_{4}(t)=\frac{1}{6} t\left(3-t^{2}\right),
\end{array}
$$

$\kappa_{i}(s)$ is the $i$ th component of $(I-[B])^{-1} \mathcal{K}(s)$,

$$
k_{0}(t, s)= \begin{cases}\frac{1}{6} t(1-s)\left(3 s-t^{2}\right), & 0 \leq t \leq s \leq 1 \\ \frac{1}{6} s\left(t^{3}-3 t^{2}+3 t-s^{2}\right), & 0 \leq s \leq t \leq 1\end{cases}
$$

We put forward the following hypotheses: 
$\left(C_{2}\right) B_{i}$ is of bounded variation and $\mathcal{K}_{i}(s) \geq 0, \forall s \in[0,1](i=1,2,3,4)$;

$\left(C_{3}\right)$ The $4 \times 4$ matrix $[B]$ is positive and its $(i, j)$ th entry is $\beta_{i}\left[\gamma_{j}\right]$, i.e., it has nonnegative entries. In addition, its spectrum radius $r([B])<1$.

Remark The integral operator $S$ in (2.1) and the corresponding Green's function $k_{S}(t, s)$ in (2.2) are obtained completely following the method in Webb and Infante [16, pp. 241-243] though $f$ is independent of the derivatives of $u$ there.

Lemma 2.1 If $\left(C_{2}\right)$ and $\left(C_{3}\right)$ hold, then $\kappa_{i}(s) \geq 0(i=1,2,3,4)$ and, for $t, s \in[0,1]$,

$$
c_{0}(t) \Phi_{0}(s) \leq k_{S}(t, s) \leq \Phi_{0}(s)
$$

where

$$
\Phi_{0}(s)=\sum_{i=1}^{4} \kappa_{i}(s)+\frac{1}{6} s(1-s)(1+s), \quad c_{0}(t)=\frac{1}{6} t\left(t^{2}-3 t+3\right)
$$

and

$$
c_{1}(t) \Phi_{1}(s) \leq \frac{\partial k_{S}(t, s)}{\partial t} \leq \Phi_{1}(s), \quad c_{2}(t) \Phi_{2}(s) \leq-\frac{\partial^{2} k_{S}(t, s)}{\partial t^{2}} \leq \Phi_{2}(s)
$$

where

$$
\begin{array}{ll}
\Phi_{1}(s)=\sum_{i=2}^{4} \kappa_{i}(s)+\frac{1}{2} s(1-s), & c_{1}(t)=\frac{1}{2}\left(1-t^{2}\right), \\
\Phi_{2}(s)=\sum_{i=3}^{4} \kappa_{i}(s)+s(1-s), & c_{2}(t)=\min \{t, 1-t\} .
\end{array}
$$

Proof For $s \in[0,1], \kappa_{i}(s) \geq 0(i=1,2,3,4)$ are due to [16, proof of Theorem 2.4] since both $(I-[B])^{-1}$ and $\mathcal{K}(s)$ are nonnegative.

According to the following two inequalities:

$$
\begin{aligned}
& \frac{1}{6} t\left(t^{2}-3 t+3\right) \sum_{i=1}^{4} \kappa_{i}(s) \leq \sum_{i=1}^{4} \kappa_{i}(s) \gamma_{i}(t) \leq \sum_{i=1}^{4} \kappa_{i}(s) \\
& \frac{1}{6} t\left(t^{2}-3 t+3\right) \frac{1}{6} s(1-s)(1+s) \\
& \leq \frac{1}{2} t\left(3-t^{2}\right) \frac{1}{6} s(1-s)(1+s) \\
& \leq k_{0}(t, s) \leq \frac{1}{6} s(1-s)(1+s)
\end{aligned}
$$

we have, for $t, s \in[0,1]$,

$$
c_{0}(t) \Phi_{0}(s) \leq k_{S}(t, s)=\sum_{i=1}^{4} \kappa_{i}(s) \gamma_{i}(t)+k_{0}(t, s) \leq \Phi_{0}(s) .
$$


Moreover, the next two inequalities

$$
\begin{aligned}
& \frac{1}{2}(1-t)^{2} \sum_{i=2}^{4} \kappa_{i}(s) \leq \sum_{i=1}^{4} \kappa_{i}(s) \gamma_{i}^{\prime}(t) \leq \sum_{i=2}^{4} \kappa_{i}(s) \\
& \frac{1}{2}(1-t)^{2} \frac{1}{2} s(2-s) \leq(1-t)^{2} \frac{1}{2} s(2-s) \leq \frac{\partial k_{0}(t, s)}{\partial t} \leq \frac{1}{2} s(1-s)
\end{aligned}
$$

imply, for $t, s \in[0,1]$,

$$
c_{1}(t) \Phi_{1}(s) \leq \frac{\partial k_{S}(t, s)}{\partial t}=\sum_{i=1}^{4} \kappa_{i}(s) \gamma_{i}^{\prime}(t)+\frac{\partial k_{0}(t, s)}{\partial t} \leq \Phi_{1}(s) .
$$

Finally from the two inequalities

$$
\begin{aligned}
\min \{t, 1-t\} \sum_{i=3}^{4} \kappa_{i}(s) & \leq-\sum_{i=1}^{4} \kappa_{i}(s) \gamma_{i}^{\prime \prime}(t) \\
& =(1-t) \kappa_{3}(s)+t \kappa_{4}(s) \leq \sum_{i=3}^{4} \kappa_{i}(s), \\
\min \{t, 1-t\} s(1-s) \leq & -\frac{\partial^{2} k_{0}(t, s)}{\partial t^{2}} \leq s(1-s)
\end{aligned}
$$

it follows that

$$
c_{2}(t) \Phi_{2}(s) \leq-\frac{\partial^{2} k_{S}(t, s)}{\partial t^{2}}=-\sum_{i=1}^{4} \kappa_{i}(s) \gamma_{i}^{\prime \prime}(t)-\frac{\partial^{2} k_{0}(t, s)}{\partial t^{2}} \leq \Phi_{2}(s)
$$

for $t, s \in[0,1]$.

Define the subsets in $C^{2}[0,1]$ as follows:

$$
\begin{aligned}
P= & \left\{u \in C^{2}[0,1]: u(t) \geq 0, u^{\prime}(t) \geq 0, u^{\prime \prime}(t) \leq 0, \forall t \in[0,1]\right\}, \\
K= & \left\{u \in P: u(t) \geq c_{0}(t)\|u\|_{C}, u^{\prime}(t) \geq c_{1}(t)\left\|u^{\prime}\right\|_{C^{\prime}}\right. \\
& \left.-u^{\prime \prime}(t) \geq c_{2}(t)\left\|u^{\prime \prime}\right\|_{C}, \forall t \in[0,1] ; \beta_{i}[u] \geq 0(i=1,2,3,4)\right\} .
\end{aligned}
$$

Clearly both $P$ and $K$ are cones, and it is easy to check that $P$ is a solid cone. Denote the cone ordering induced by $P, u \preceq v$ for $u, v \in X$ if and only if $v-u \in P$ and equivalently $v \succeq u$.

Now we define linear operators in $C^{2}[0,1]$ :

$$
\begin{aligned}
& \left(L_{i} u\right)(t)=\int_{0}^{1} k_{S}(t, s)\left(a_{i} u(s)+b_{i} u^{\prime}(s)-c_{i} u^{\prime \prime}(s)\right) d s \quad(i=1,2), \\
& \left(L_{3} u\right)(t)=a_{1} \int_{0}^{1} k_{S}(t, s) u(s) d s,
\end{aligned}
$$

where $a_{i}, b_{i}, c_{i}(i=1,2)$ are nonnegative constants.

Similar to [16], we have the following Lemma 2.2 by Lemma 2.1 . 
Lemma 2.2 If $\left(C_{1}\right)-\left(C_{3}\right)$ hold, then $S: P \rightarrow K$ and $L_{i}: C^{2}[0,1] \rightarrow C^{2}[0,1]$ are completely continuous operators with $L_{i}(P) \subset K(i=1,2,3)$.

Theorem 2.1 Under hypotheses $\left(C_{1}\right)-\left(C_{3}\right)$ suppose that

$\left(F_{1}\right)$ there exist constants $a_{2}, b_{2}, c_{2} \geq 0$ and $r>0$ such that

$$
f\left(t, x_{1}, x_{2}, x_{3}\right) \leq a_{2} x_{1}+b_{2} x_{2}-c_{2} x_{3}
$$

for all $\left(t, x_{1}, x_{2}, x_{3}\right) \in[0,1] \times[0, r]^{2} \times[-r, 0] ;$ moreover, the spectral radius $r\left(L_{2}\right)<1$, where $L_{2}$ is defined by (2.7),

$\left(F_{2}\right)$ there exist positive constants $a_{1}, b_{1}, c_{1}, C_{0}$ satisfying

$$
\begin{aligned}
& \min \left\{\frac{a_{1}}{6} \int_{0}^{1} c_{0}(s) \Phi_{0}(s) d s, \frac{b_{1}}{2} \int_{0}^{1} c_{1}(s) \Phi_{1}(s) d s,\right. \\
& \left.\frac{c_{1}}{2} \int_{0}^{1}\left(\kappa_{3}(s)+\kappa_{4}(s)\right) c_{2}(s) d s\right\}>1
\end{aligned}
$$

such that

$$
f\left(t, x_{1}, x_{2}, x_{3}\right) \geq a_{1} x_{1}+b_{1} x_{2}-c_{1} x_{3}-C_{0}
$$

for all $\left(t, x_{1}, x_{2}, x_{3}\right) \in[0,1] \times \mathbb{R}_{+}^{2} \times \mathbb{R}_{-}$.

Then BVP (1.1) has one positive solution in $K$.

Proof (i) First we prove that $\mu S u \neq u$ for $u \in K \cap \partial \Omega_{r}$ and $\mu \in[0,1]$, where $\Omega_{r}=\{u \in$ $\left.C^{2}[0,1]:\|u\|_{C^{2}}<r\right\}$.

In fact, if there exist $u_{1} \in K \cap \partial \Omega_{r}$ and $\mu_{0} \in[0,1]$ such that $u_{1}=\mu_{0} S u_{1}$, then we deduce from

$$
0 \leq u_{1}(t), u_{1}^{\prime}(t) \leq r, \quad 0 \leq-u_{1}^{\prime \prime}(t) \leq r, \quad \forall t \in[0,1]
$$

and (2.9) that, for $t \in[0,1]$,

$$
u_{1}(t) \leq\left(L_{2} u_{1}\right)(t), \quad u_{1}^{\prime}(t) \leq\left(L_{2} u_{1}\right)^{\prime}(t), \quad u_{1}^{\prime \prime}(t) \geq\left(L_{2} u_{1}\right)^{\prime \prime}(t)
$$

thus $\left(I-L_{2}\right) u_{1} \preceq 0$. Because of the spectral radius $r\left(L_{2}\right)<1$, we know that $I-L_{2}$ has a bounded inverse operator $\left(I-L_{2}\right)^{-1}: P \rightarrow P$ and $u_{1} \preceq\left(I-L_{2}\right)^{-1} 0=0$, which contradicts $u_{1} \in K \cap \partial \Omega_{r}$.

Therefore, $i\left(S, K \cap \Omega_{r}, K\right)=1$ follows from Lemma 1.1.

(ii) In this step we construct a homotopy and find a subset $\Omega_{R}$ in order to compute the fixed point index later.

Let $M$ be

$$
\begin{aligned}
& \max \left\{\frac{6 C_{0} \int_{0}^{1} \Phi_{0}(s) d s}{a_{1} \int_{0}^{1} c_{0}(s) \Phi_{0}(s) d s-6}, \frac{2 C_{0} \int_{0}^{1} \Phi_{1}(s) d s}{b_{1} \int_{0}^{1} c_{1}(s) \Phi_{1}(s) d s-2},\right. \\
& \left.\frac{C_{0} \int_{0}^{1}\left(\kappa_{3}(s)+\kappa_{4}(s)\right) d s}{c_{1} \int_{0}^{1}\left(\kappa_{3}(s)+\kappa_{4}(s)\right) c_{2}(s) d s-2}\right\} .
\end{aligned}
$$


Obviously, $M>0$ if we notice that

$$
C_{0} \int_{0}^{1}\left(\kappa_{3}(s)+\kappa_{4}(s)\right) d s>0
$$

since $\frac{c_{1}}{2} \int_{0}^{1}\left(\kappa_{3}(s)+\kappa_{4}(s)\right) c_{2}(s) d s>1$ by $(2.10)$.

For $u \in K$, define the homotopy $H(\lambda, u)=S u+\lambda v$, where

$$
v(t)=C_{0} \int_{0}^{1} k_{S}(t, s) d s
$$

then $v \in K$ and $H:[0,1] \times K \rightarrow K$ is completely continuous.

Let $R>\max \{r, M\}$ and we will show that

$$
H(\lambda, u) \neq u, \quad \forall u \in K \cap \partial \Omega_{R}, \lambda \in[0,1],
$$

where $\Omega_{R}=\left\{u \in C^{2}[0,1]:\|u\|_{C^{2}}<R\right\}$.

If it does not hold, there exist $u_{2} \in K \cap \partial \Omega_{R}$ and $\lambda_{0} \in[0,1]$ such that

$$
H\left(\lambda_{0}, u_{2}\right)=u_{2},
$$

thus (2.11) and Lemma 2.1 yield that

$$
\begin{aligned}
\left\|u_{2}\right\|_{C} & =u_{2}(1) \\
& =\int_{0}^{1} k_{S}(1, s) f\left(s, u_{2}(s), u_{2}^{\prime}(s), u_{2}^{\prime \prime}(s)\right) d s+\lambda_{0} C_{0} \int_{0}^{1} k_{S}(1, s) d s \\
& \geq \int_{0}^{1} k_{S}(1, s)\left[a_{1} u_{2}(s)+b_{1} u_{2}^{\prime}(s)-c_{1} u_{2}^{\prime \prime}(s)-C_{0}+\lambda_{0} C_{0}\right] d s \\
& \geq \int_{0}^{1} k_{S}(1, s)\left[a_{1} u_{2}(s)-C_{0}\right] d s \\
& \geq \frac{a_{1}}{6} \int_{0}^{1} \Phi_{0}(s) u_{2}(s) d s-C_{0} \int_{0}^{1} \Phi_{0}(s) d s \\
& \geq \frac{a_{1}}{6}\left\|u_{2}\right\|_{C} \int_{0}^{1} c_{0}(s) \Phi_{0}(s) d s-C_{0} \int_{0}^{1} \Phi_{0}(s) d s, \\
\left\|u_{2}^{\prime}\right\|_{C} & =u_{2}^{\prime}(0) \\
& =\int_{0}^{1} \frac{\partial k_{S}(0, s)}{\partial t} f\left(s, u_{2}(s), u_{2}^{\prime}(s), u_{2}^{\prime \prime}(s)\right) d s+\lambda_{0} C_{0} \int_{0}^{1} \frac{\partial k_{S}(0, s)}{\partial t} d s \\
& \geq \int_{0}^{1} \frac{\partial k_{S}(0, s)}{\partial t}\left[a_{1} u_{2}(s)+b_{1} u_{2}^{\prime}(s)-c_{1} u_{2}^{\prime \prime}(s)-C_{0}+\lambda_{0} C_{0}\right] d s \\
& \geq \int_{0}^{1} \frac{\partial k_{S}(0, s)}{\partial t}\left[b_{1} u_{2}^{\prime}(s)-C_{0}\right] d s \\
& \frac{b_{1}}{2} \int_{0}^{1} \Phi_{1}(s) u_{2}^{\prime}(s) d s-C_{0} \int_{0}^{1} \Phi_{1}(s) d s \\
& \\
&
\end{aligned}
$$


Since $u_{2}^{(4)}(t)=f\left(t, u_{2}(t), u_{2}^{\prime}(t), u_{2}^{\prime \prime}(t)\right)+\lambda_{0} C_{0} \geq 0$ for $t \in[0,1]$, we know that if $0 \leq u^{\prime \prime \prime}(0) \leq$ $u^{\prime \prime \prime}(1)$, then

$$
\left\|u^{\prime \prime}\right\|_{C}=-u_{2}^{\prime \prime}(0) \geq-\frac{1}{2}\left(u_{2}^{\prime \prime}(0)+u_{2}^{\prime \prime}(1)\right)
$$

if $u^{\prime \prime \prime}(0) \leq u^{\prime \prime \prime}(1) \leq 0$, then

$$
\left\|u^{\prime \prime}\right\|_{C}=-u_{2}^{\prime \prime}(1) \geq-\frac{1}{2}\left(u_{2}^{\prime \prime}(0)+u_{2}^{\prime \prime}(1)\right)
$$

if $u^{\prime \prime \prime}(0) \leq 0 \leq u^{\prime \prime \prime}(1)$, then there exists $\xi \in[0,1]$ such that

$$
\left\|u_{2}^{\prime \prime}\right\|_{C}=-u_{2}^{\prime \prime}(\xi) \geq-\frac{1}{2}\left(u_{2}^{\prime \prime}(0)+u_{2}^{\prime \prime}(1)\right)
$$

Therefore the proof of Lemma 2.1 leads to

$$
\begin{aligned}
\left\|u_{2}^{\prime \prime}\right\|_{C} & \geq-\frac{1}{2}\left(u_{2}^{\prime \prime}(0)+u_{2}^{\prime \prime}(1)\right) \\
& =-\frac{1}{2} \int_{0}^{1}\left(\frac{\partial^{2} k_{S}(0, s)}{\partial t^{2}}+\frac{\partial^{2} k_{S}(1, s)}{\partial t^{2}}\right)\left[f\left(s, u_{2}(s), u_{2}^{\prime}(s), u_{2}^{\prime \prime}(s)\right)+\lambda_{0} C_{0}\right] d s \\
& =\frac{1}{2} \int_{0}^{1}\left(\kappa_{3}(s)+\kappa_{4}(s)\right)\left[f\left(s, u_{2}(s), u_{2}^{\prime}(s), u_{2}^{\prime \prime}(s)\right)+\lambda_{0} C_{0}\right] d s \\
& \geq \frac{1}{2} \int_{0}^{1}\left(\kappa_{3}(s)+\kappa_{4}(s)\right)\left[a_{1} u_{2}(s)+b_{1} u_{2}^{\prime}(s)-c_{1} u_{2}^{\prime \prime}(s)-C_{0}+\lambda_{0} C_{0}\right] d s \\
& \geq \frac{1}{2} \int_{0}^{1}\left(\kappa_{3}(s)+\kappa_{4}(s)\right)\left[-c_{1} u_{2}^{\prime \prime}(s)-C_{0}\right] d s \\
& \geq \frac{c_{1}}{2}\left\|u_{2}^{\prime \prime}\right\|_{C} \int_{0}^{1}\left(\kappa_{3}(s)+\kappa_{4}(s)\right) c_{2}(s) d s-\frac{C_{0}}{2} \int_{0}^{1}\left(\kappa_{3}(s)+\kappa_{4}(s)\right) d s,
\end{aligned}
$$

which implies by (2.12), (2.15), and (2.16) that

$$
\left\|u_{2}\right\|_{C} \leq M, \quad\left\|u_{2}^{\prime}\right\|_{C} \leq M, \quad\left\|u_{2}^{\prime \prime}\right\|_{C} \leq M,
$$

a contradiction to $\left\|u_{2}\right\|_{C^{2}}=R>M$.

From (2.13) it follows that

$$
i\left(S, K \cap \Omega_{R}, K\right)=i\left(H(0, \cdot), K \cap \Omega_{R}, K\right)=i\left(H(1, \cdot), K \cap \Omega_{R}, K\right)
$$

by the homotopy invariance property of fixed point index.

(iii) Now we search for an appropriate element in $K$ for the sake of the next step. For the function $c_{0}(t)=\frac{1}{6} t\left(t^{2}-3 t+3\right)$, we have from (2.8) and Lemma 2.1 that

$$
\left(L_{3} c_{0}\right)(t)=a_{1} \int_{0}^{1} k_{S}(t, s) c_{0}(s) d s \geq\left(a_{1} \int_{0}^{1} c_{0}(s) \Phi_{0}(s) d s\right) c_{0}(t)
$$

From (2.10) it follows that $a_{1} \int_{0}^{1} c_{0}(s) \Phi_{0}(s) d s>6$. Since $L_{3}$ is a completely continuous linear operator in $C[0,1]$, we consider the nonnegative cone $C^{+}[0,1]=\{u \in C[0,1]: u(t) \geq 0, \forall t \in$ 
$[0,1]\}$ in Lemma 1.4. There exist $\lambda_{1}>6$ and $\varphi_{0} \in C^{+}[0,1] \backslash\{0\}$ such that $\varphi_{0}=\lambda_{1}^{-1} L_{3} \varphi_{0}$. Obviously $\varphi_{0} \in P$ can be directly verified, and thus $\varphi_{0} \in K$ by Lemma 2.2 .

(iv) Now we prove that $u-H(1, u) \neq v \varphi_{0}$ for $u \in K \cap \partial \Omega_{R}$ and $v \geq 0$, where $\varphi_{0}$ is as in step (iii), and hence

$$
i\left(H(1, \cdot), K \cap \Omega_{R}, K\right)=0
$$

holds by Lemma 1.2.

If there exist $u_{0} \in K \cap \partial \Omega_{R}$ and $v_{0} \geq 0$ such that $u_{0}-H\left(1, u_{0}\right)=v_{0} \varphi_{0}$. Clearly $v_{0}>0$ by (2.13), and thus

$$
u_{0}(t)=\left(H\left(1, u_{0}\right)\right)(t)+v_{0} \varphi_{0}(t) \geq v_{0} \varphi_{0}(t)
$$

for $t \in[0,1]$. Set

$$
v^{*}=\sup \left\{v>0: u_{0}(t) \geq v \varphi_{0}(t), \forall t \in[0,1]\right\},
$$

then $v_{0} \leq v^{*}<+\infty$ and $u_{0}(t) \geq v^{*} \varphi_{0}(t)$ for $t \in[0,1]$. From (2.11) we have that, for $t \in[0,1]$,

$$
\begin{aligned}
u_{0}(t) & =\left(H\left(1, u_{0}\right)\right)(t)+v_{0} \varphi_{0}(t) \geq\left(L_{3} u_{0}\right)(t)+v_{0} \varphi_{0}(t) \\
& \geq v^{*}\left(L_{3} \varphi_{0}\right)(t)+v_{0} \varphi_{0}(t)=\lambda_{1} v^{*} \varphi_{0}(t)+v_{0} \varphi_{0}(t) .
\end{aligned}
$$

Since $\lambda_{1}>6$, we have $\lambda_{1} v^{*}+v_{0}>v^{*}$, which contradicts the definition of $v^{*}$.

(vi) From (2.17) and (2.18) it follows that $i\left(S, K \cap \Omega_{R}, K\right)=0$ and

$$
i\left(S, K \cap\left(\Omega_{R} \backslash \bar{\Omega}_{r}\right), K\right)=i\left(S, K \cap \Omega_{R}, K\right)-i\left(S, K \cap \Omega_{r}, K\right)=-1 .
$$

Hence $S$ has one fixed solution, i.e., BVP (1.1) has one positive solution in $K$.

Theorem 2.2 Under hypotheses $\left(C_{1}\right)-\left(C_{3}\right)$ suppose that

$\left(F_{3}\right)$ there exist constants $a_{1}, b_{1}, c_{1}, C_{0} \geq 0$ such that

$$
f\left(t, x_{1}, x_{2}, x_{3}\right) \leq a_{1} x_{1}+b_{1} x_{2}-c_{1} x_{3}+C_{0}
$$

for all $\left(t, x_{1}, x_{2}, x_{3}\right) \in[0,1] \times \mathbb{R}_{+}^{2} \times \mathbb{R}_{-}$, moreover the spectral radius $r\left(L_{1}\right)<1$;

$\left(F_{4}\right)$ there exist constants $a_{2}, b_{2}, c_{2} \geq 0$ and $r>0$ such that

$$
f\left(t, x_{1}, x_{2}, x_{3}\right) \geq a_{2} x_{1}+b_{2} x_{2}-c_{2} x_{3}
$$

for all $\left(t, x_{1}, x_{2}, x_{3}\right) \in[0,1] \times[0, r]^{2} \times[-r, 0]$, moreover the spectral radius $r\left(L_{2}\right) \geq 1$; where $L_{i}: C^{2}[0,1] \rightarrow C^{2}[0,1](i=1,2)$ are defined by $(2.7)$.

Then BVP (1.1) has one positive solution in $K$.

Proof Let $W=\{u \in K$ : there exists a $\mu \in[0,1]$ with $u=\mu S u\}$ where $S$ and $K$ are respectively defined in (2.1) and (2.6). 
We first assert that $W$ is a bounded set. In fact, if $u \in W$, then $u=\mu S u$ for some $\mu \in[0,1]$. From (2.7) and (2.19) we have that

$$
\begin{aligned}
u(t) & =\mu(S u)(t)=\mu \int_{0}^{1} k_{S}(t, s) f\left(s, u(s), u^{\prime}(s), u^{\prime \prime}(s)\right) d s \\
& \leq \int_{0}^{1} k_{S}(t, s)\left[a_{1} u(s)+b_{1} u^{\prime}(s)-c_{1} u^{\prime \prime}(s)+C_{0}\right] d s \\
& =\left(L_{1} u\right)(t)+C_{0} \int_{0}^{1} k_{S}(t, s) d s
\end{aligned}
$$

and

$$
\left(\left(I-L_{1}\right) u\right)(t) \leq C_{0} \int_{0}^{1} k_{S}(t, s) d s=: v(t), \quad t \in[0,1]
$$

Obviously $v \in P$ and it is easy to see from (2.19) that, for $t \in[0,1]$,

$$
u^{\prime}(t) \leq\left(L_{1} u\right)^{\prime}(t)+v^{\prime}(t), \quad u^{\prime \prime}(t) \geq\left(L_{1} u\right)^{\prime \prime}(t)+v^{\prime \prime}(t)
$$

thus $\left(I-L_{1}\right) u \preceq v$. Because of the spectral radius $r\left(L_{1}\right)<1$, we know that $I-L_{1}$ has a bounded inverse operator $\left(I-L_{1}\right)^{-1}$, which can be written as

$$
\left(I-L_{1}\right)^{-1}=I+L_{1}+L_{1}^{2}+\cdots+L_{1}^{n}+\cdots .
$$

Since $L_{1}(P) \subset K \subset P$ by Lemma 2.2, we have $\left(I-L_{1}\right)^{-1}(P) \subset P$, which implies the inequality $u \preceq\left(I-L_{1}\right)^{-1} v$. Therefore, for $t \in[0,1]$,

$$
\begin{aligned}
& 0 \leq u(t) \leq\left(\left(I-L_{1}\right)^{-1} v\right)(t), \quad 0 \leq u^{\prime}(t) \leq\left(\left(I-L_{1}\right)^{-1} v\right)^{\prime}(t), \\
& 0 \geq u^{\prime \prime}(t) \geq\left(\left(I-L_{1}\right)^{-1} v\right)^{\prime \prime}(t),
\end{aligned}
$$

and hence $\|u\|_{C^{2}} \leq\left\|\left(I-L_{1}\right)^{-1} v\right\|_{C^{2}}$, i.e., $W$ is bounded.

Now select $R>\max \{r$, sup $W\}$, then $\mu S u \neq u$ for $u \in K \cap \partial \Omega_{R}$ and $\mu \in[0,1]$, and $i(S, K \cap$ $\left.\Omega_{R}, K\right)=1$ follows from Lemma 1.1 .

Since $L_{2}: P \rightarrow K \subset P$ and $r\left(L_{2}\right) \geq 1$, it follows from Lemma 1.3 that there exists $\varphi_{0} \in$ $P \backslash\{0\}$ such that $L_{2} \varphi_{0}=r\left(L_{2}\right) \varphi_{0}$. Furthermore, $\varphi_{0}=\left(r\left(L_{2}\right)\right)^{-1} L_{2} \varphi_{0} \in K$.

We may suppose that $S$ has no fixed points in $K \cap \partial \Omega_{r}$ and will show that $u-S u \neq v \varphi_{0}$ for $u \in K \cap \partial \Omega_{r}$ and $v \geq 0$.

Otherwise, there exist $u_{0} \in K \cap \partial \Omega_{r}$ and $v_{0} \geq 0$ such that $u_{0}-S u_{0}=v_{0} \varphi_{0}$, and it is clear that $v_{0}>0$. Since $u_{0} \in K \cap \partial \Omega_{r}$, we have

$$
0 \leq u_{0}(t), u_{0}^{\prime}(t) \leq r, \quad-r \leq u_{0}^{\prime \prime}(t) \leq 0, \quad \forall t \in[0,1]
$$

It follows from (2.2), (2.7), and (2.20) that $\forall t \in[0,1]$,

$$
\left(S u_{0}\right)(t) \geq\left(L_{2} u_{0}\right)(t), \quad\left(S u_{0}\right)^{\prime}(t) \geq\left(L_{2} u_{0}\right)^{\prime}(t), \quad\left(S u_{0}\right)^{\prime \prime}(t) \leq\left(L_{2} u_{0}\right)^{\prime \prime}(t),
$$


which imply that

$$
u_{0}=v_{0} \varphi_{0}+S u_{0} \succeq v_{0} \varphi_{0}+L_{2} u_{0} \succeq v_{0} \varphi_{0}
$$

Set $v^{*}=\sup \left\{v>0: u_{0} \succeq v \varphi_{0}\right\}$, then $v_{0} \leq v^{*}<+\infty$ and $u_{0} \succeq v^{*} \varphi_{0}$. Thus it follows from (2.21) that

$$
u_{0} \succeq v_{0} \varphi_{0}+L_{2} u_{0} \succeq v_{0} \varphi_{0}+v^{*} L_{2} \varphi_{0}=v_{0} \varphi_{0}+v^{*} r\left(L_{2}\right) \varphi_{0} .
$$

But $r\left(L_{2}\right) \geq 1$, so $u_{0} \succeq\left(v_{0}+v^{*}\right) \varphi_{0}$, which is a contradiction to the definition of $v^{*}$. Therefore $u-S u \neq v \varphi_{0}$ for $u \in K \cap \partial \Omega_{r}$ and $v \geq 0$.

From Lemma 1.2 it follows that $i\left(S, K \cap \Omega_{r}, K\right)=0$.

Making use of the properties of fixed point index, we have that

$$
i\left(S, K \cap\left(\Omega_{R} \backslash \bar{\Omega}_{r}\right), K\right)=i\left(S, K \cap \Omega_{R}, K\right)-i\left(S, K \cap \Omega_{r}, K\right)=1,
$$

and hence $S$ has one fixed point in $K$. Therefore, BVP (1.1) has one positive solution in $K$.

As an example, we consider the fourth-order boundary problem under mixed multipoint and integral boundary conditions with sign-changing coefficients:

$$
\left\{\begin{array}{l}
u^{(4)}(t)=f\left(t, u(t), u^{\prime}(t), u^{\prime \prime}(t)\right), \quad t \in[0,1], \\
u(0)=\frac{1}{4} u\left(\frac{1}{4}\right)-\frac{1}{12} u\left(\frac{3}{4}\right), \quad u^{\prime}(1)=\int_{0}^{1} u(t)\left(t-\frac{1}{8}\right) d t, \\
u^{\prime \prime}(0)+\frac{1}{2} u\left(\frac{1}{2}\right)-\frac{1}{4} u\left(\frac{3}{4}\right)=0, \quad u^{\prime \prime}(1)+\frac{1}{2} u\left(\frac{1}{4}\right)-\frac{1}{4} u\left(\frac{1}{2}\right)=0,
\end{array}\right.
$$

thus $\beta_{1}[u]=\frac{1}{4} u\left(\frac{1}{4}\right)-\frac{1}{12} u\left(\frac{3}{4}\right), \beta_{2}[u]=\int_{0}^{1} u(t)\left(t-\frac{1}{8}\right) d t, \beta_{3}[u]=\frac{1}{2} u\left(\frac{1}{2}\right)-\frac{1}{4} u\left(\frac{3}{4}\right)$, and $\beta_{4}[u]=$ $\frac{1}{2} u\left(\frac{1}{4}\right)-\frac{1}{4} u\left(\frac{1}{2}\right)$. We estimate some coefficients, and Matlab is used in some places.

$$
\begin{aligned}
\mathcal{K}_{1}(s) & =\int_{0}^{1} k_{0}(t, s) d B_{1}(t)=\frac{1}{4} k_{0}\left(\frac{1}{4}, s\right)-\frac{1}{12} k_{0}\left(\frac{3}{4}, s\right) \\
& = \begin{cases}-\frac{1}{36} s^{3}+\frac{1}{96} s, & 0 \leq s \leq \frac{1}{4}, \\
\frac{1}{72} s^{3}-\frac{1}{32} s^{2}+\frac{7}{384} s-\frac{1}{1536}, & \frac{1}{4}<s \leq \frac{3}{4}, \\
-\frac{1}{192} s+\frac{1}{192}, & \frac{3}{4}<s \leq 1,\end{cases}
\end{aligned}
$$

and hence $0 \leq \mathcal{K}_{1}(s)<0.0026$;

$$
\mathcal{K}_{2}(s)=\int_{0}^{1} k_{0}(t, s)\left(t-\frac{1}{8}\right) d t=\frac{1}{120} s^{5}-\frac{1}{192} s^{4}-\frac{1}{16} s^{3}+\frac{57}{960} s \quad(0 \leq s \leq 1),
$$

and hence $0 \leq \mathcal{K}_{2}(s)<0.0223$;

$$
\begin{aligned}
\mathcal{K}_{3}(s) & =\int_{0}^{1} k_{0}(t, s) d B_{3}(t)=\frac{1}{2} k_{0}\left(\frac{1}{2}, s\right)-\frac{1}{4} k_{0}\left(\frac{3}{4}, s\right) \\
& = \begin{cases}-\frac{1}{24} s^{3}+\frac{49}{1536} s, & 0 \leq s \leq \frac{1}{2}, \\
\frac{1}{24} s^{3}-\frac{1}{8} s^{2}+\frac{145}{1536} s-\frac{1}{96}, & \frac{1}{2}<s \leq \frac{3}{4}, \\
-\frac{1}{32} s^{2}+\frac{37}{1536} s+\frac{11}{1536}, & \frac{3}{4}<s \leq 1,\end{cases}
\end{aligned}
$$


and hence $0 \leq \mathcal{K}_{3}(s)<0.0108$;

$$
\begin{aligned}
\mathcal{K}_{4}(s) & =\int_{0}^{1} k_{0}(t, s) d B_{4}(t)=\frac{1}{2} k_{0}\left(\frac{1}{4}, s\right)-\frac{1}{4} k_{0}\left(\frac{1}{2}, s\right) \\
& = \begin{cases}-\frac{1}{24} s^{3}+\frac{3}{256} s, & 0 \leq s \leq \frac{1}{4}, \\
\frac{1}{24} s^{3}-\frac{1}{16} s^{2}+\frac{7}{256} s-\frac{1}{768}, & \frac{1}{4}<s \leq \frac{1}{2}, \\
-\frac{1}{256} s+\frac{1}{256}, & \frac{1}{2}<s \leq 1,\end{cases}
\end{aligned}
$$

and hence $0 \leq \mathcal{K}_{4}(s)<0.0025$.

The $4 \times 4$ matrix

$$
[B]=\left(\begin{array}{llll}
\beta_{1}\left[\gamma_{1}\right] & \beta_{1}\left[\gamma_{2}\right] & \beta_{1}\left[\gamma_{3}\right] & \beta_{1}\left[\gamma_{4}\right] \\
\beta_{2}\left[\gamma_{1}\right] & \beta_{2}\left[\gamma_{2}\right] & \beta_{2}\left[\gamma_{3}\right] & \beta_{2}\left[\gamma_{4}\right] \\
\beta_{3}\left[\gamma_{1}\right] & \beta_{3}\left[\gamma_{2}\right] & \beta_{3}\left[\gamma_{3}\right] & \beta_{3}\left[\gamma_{4}\right] \\
\beta_{4}\left[\gamma_{1}\right] & \beta_{4}\left[\gamma_{2}\right] & \beta_{4}\left[\gamma_{3}\right] & \beta_{4}\left[\gamma_{4}\right]
\end{array}\right)=\left(\begin{array}{cccc}
\frac{1}{6} & 0 & \frac{1}{96} & \frac{1}{192} \\
\frac{3}{8} & \frac{13}{48} & \frac{19}{320} & \frac{103}{960} \\
\frac{1}{4} & \frac{1}{16} & \frac{49}{1536} & \frac{59}{1536} \\
\frac{1}{4} & 0 & \frac{3}{256} & \frac{1}{256}
\end{array}\right)
$$

and its spectrum radius $r([B]) \approx 0.2976<1$. Those mean that $\left(C_{2}\right)$ and $\left(C_{3}\right)$ are satisfied.

Now we take stock of some constants in Theorem 2.1 and Theorem 2.2.

$$
(I-[B])^{-1}<\left(\begin{array}{cccc}
1.2066 & 0.0012 & 0.0132 & 0.0070 \\
0.6958 & 1.3796 & 0.0941 & 0.1559 \\
0.3688 & 0.0895 & 1.0431 & 0.0519 \\
0.3073 & 0.0014 & 0.0157 & 1.0064
\end{array}\right)
$$

and

$$
(I-[B])^{-1} \mathcal{K}(s)<\left(\begin{array}{c}
0.0033 \\
0.0340 \\
0.0143 \\
0.0035
\end{array}\right)
$$

thus $k_{S}(t, s)<0.0033+0.0340 t+0.0143 \times \frac{1}{6} t\left(t^{2}-3 t+3\right)+0.0035 \times \frac{1}{6} t\left(3-t^{2}\right)+k_{0}(t, s)<$ 0.1051 . So, for $u \in C^{2}[0,1]$ and $t \in[0,1]$,

$$
\begin{aligned}
\left|\left(L_{i} u\right)(t)\right| & \leq 0.1051 \int_{0}^{1}\left(a_{i}|u(s)|+b_{i}\left|u^{\prime}(s)\right|+c_{i}\left|u^{\prime \prime}(s)\right|\right) d s \\
& \leq 0.1051\left(a_{i}+b_{i}+c_{i}\right)\|u\|_{C^{2}} \quad(i=1,2),
\end{aligned}
$$

here $L_{i}(i=1,2)$ are defined in (2.7). Since all the terms are nonnegative in the first derivative of $k_{S}(t, s)$ with respect to $t$ and they are non-positive in the second derivative of $k_{S}(t, s)$, we also have that, for $u \in C^{2}[0,1]$ and $t \in[0,1]$,

$$
\begin{aligned}
\left|\left(L_{i} u\right)^{\prime}(t)\right| & \leq 0.1680 \int_{0}^{1}\left(a_{i}|u(s)|+b_{i}\left|u^{\prime}(s)\right|+c_{i}\left|u^{\prime \prime}(s)\right|\right) d s \\
& \leq 0.1680\left(a_{i}+b_{i}+c_{i}\right)\|u\|_{C^{2}} \quad(i=1,2),
\end{aligned}
$$




$$
\begin{aligned}
\left|\left(L_{i} u\right)^{\prime \prime}(t)\right| & \leq 0.2590 \int_{0}^{1}\left(a_{i}|u(s)|+b_{i}\left|u^{\prime}(s)\right|+c_{i}\left|u^{\prime \prime}(s)\right|\right) d s \\
& \leq 0.2590\left(a_{i}+b_{i}+c_{i}\right)\|u\|_{C^{2}} \quad(i=1,2) .
\end{aligned}
$$

Therefore the radius $r\left(L_{i}\right) \leq\left\|L_{i}\right\| \leq 0.2590\left(a_{i}+b_{i}+c_{i}\right)<1$ if

$$
a_{i}+b_{i}+c_{i}<0.2590^{-1} \quad(i=1,2)
$$

On the other hand, we have from Lemma 2.1 and Lemma 2.2 that, for $u \in K \backslash\{0\}$ and $t \in[0,1]$,

$$
\begin{aligned}
\left(L_{2} u\right)(t) & \geq \int_{0}^{1} k_{S}(t, s) a_{2} u(s) d s \geq a_{2} c_{0}(t) \int_{0}^{1} \Phi_{0}(s) u(s) d s \\
& \geq a_{2} c_{0}(t) \int_{0}^{1} \Phi_{0}(s) c_{0}(s)\|u\|_{C} d s=a_{2} c_{0}(t)\|u\|_{C} \int_{0}^{1} c_{0}(s) \Phi_{0}(s) d s
\end{aligned}
$$

and

$$
\left\|\left(L_{2} u\right)\right\|_{C}=\left(L_{2} u\right)(1) \geq \frac{1}{6} a_{2}\|u\|_{C} \int_{0}^{1} c_{0}(s) \Phi_{0}(s) d s,
$$

hence

$$
\begin{aligned}
\left(L_{2}^{2} u\right)(t) & \geq a_{2} \int_{0}^{1} k_{S}(t, s)\left(L_{2} u\right)(s) d s \\
& \geq a_{2} c_{0}(t) \int_{0}^{1} \Phi_{0}(s)\left(L_{2} u\right)(s) d s \geq a_{2} c_{0}(t) \int_{0}^{1} \Phi_{0}(s) c_{0}(s)\left\|\left(L_{2} u\right)\right\|_{C} d s \\
& \geq \frac{1}{6} a_{2}^{2} c_{0}(t)\|u\|_{C}\left(\int_{0}^{1} c_{0}(s) \Phi_{0}(s) d s\right)^{2}
\end{aligned}
$$

and

$$
\left\|\left(L_{2}^{2} u\right)\right\|_{C}=\left(L_{2}^{2} u\right)(1) \geq \frac{1}{36} a_{2}^{2}\|u\|_{C}\left(\int_{0}^{1} c_{0}(s) \Phi_{0}(s) d s\right)^{2} .
$$

By induction,

$$
\left\|\left(L_{2}^{n} u\right)\right\|_{C}=\left(L_{2}^{n} u\right)(1) \geq\left(\frac{a_{2}}{6}\right)^{n}\|u\|_{C}\left(\int_{0}^{1} c_{0}(s) \Phi_{0}(s) d s\right)^{n} .
$$

As a result, it follows that, for $u \in K \backslash\{0\}$,

$$
\left\|L_{2}^{n}\right\|\|u\|_{C^{2}} \geq\left\|L_{2}^{n} u\right\|_{C^{2}} \geq\left\|L_{2}^{n} u\right\|_{C} \geq\left(\frac{a_{2}}{6}\right)^{n}\|u\|_{C}\left(\int_{0}^{1} c_{0}(s) \Phi_{0}(s) d s\right)^{n},
$$

and according to Gelfand's formula, the spectral radius

$$
\begin{aligned}
r\left(L_{2}\right) & =\lim _{n \rightarrow \infty}\left\|L_{2}^{n}\right\|^{1 / n} \\
& \geq \frac{a_{2}}{6}\left(\int_{0}^{1} c_{0}(s) \Phi_{0}(s) d s\right) \lim _{n \rightarrow \infty}\left(\frac{\|u\|_{C}}{\|u\|_{C^{2}}}\right)^{1 / n}=\frac{a_{2}}{6}\left(\int_{0}^{1} c_{0}(s) \Phi_{0}(s) d s\right),
\end{aligned}
$$


which implies that $r\left(L_{2}\right) \geq 1$ when

$$
\begin{aligned}
a_{2} & \geq \frac{30240}{29}=\frac{6}{\int_{0}^{1} \frac{1}{6} s(1-s)(1+s) \times \frac{1}{6} s\left(s^{2}-3 s+3\right) d s} \\
& \geq \frac{6}{\int_{0}^{1} c_{0}(s) \Phi_{0}(s) d s} .
\end{aligned}
$$

Example 2.1 If

$$
f\left(t, x_{1}, x_{2}, x_{3}\right)=\frac{x_{1}^{5}+x_{2}^{5}-x_{3}^{5}}{1+x_{1}^{2}+x_{2}^{2}+x_{3}^{2}},
$$

then BVP (2.22) has a positive solution.

Proof Take $a_{2}=b_{2}=c_{2}=1, r<1$, it is easy to check that (2.9) and (2.23) for $i=2$ are satisfied. Now take $a_{1}=1043, b_{1}=69, c_{1}=903$, it is clear that

$$
\begin{aligned}
\frac{a_{1}}{6} \int_{0}^{1} c_{0}(s) \Phi_{0}(s) d s & =\frac{a_{1}}{36} \int_{0}^{1} s\left(s^{2}-3 s+3\right) \Phi_{0}(s) d s \\
& >\frac{a_{1}}{36} \int_{0}^{1} s\left(s^{2}-3 s+3\right) \frac{1}{6} s(1-s)(1+s) d s \\
& >\frac{30240}{29} \times \frac{1}{216} \int_{0}^{1} s^{2}\left(s^{2}-3 s+3\right)(1-s)(1+s) d s=1, \\
\frac{b_{1}}{2} \int_{0}^{1} c_{1}(s) \Phi_{1}(s) d s & =\frac{b_{1}}{4} \int_{0}^{1}\left(1-s^{2}\right) \Phi_{1}(s) d s \\
& >\frac{b_{1}}{4} \int_{0}^{1}\left(1-s^{2}\right) \frac{1}{2} s(1-s) d s \\
& >\frac{480}{7} \times \frac{1}{8} \int_{0}^{1}\left(1-s^{2}\right) s(1-s) d s=1 .
\end{aligned}
$$

We also have

$$
(I-[B])^{-1}>\left(\begin{array}{llll}
1.2064 & 0.0010 & 0.0130 & 0.0068 \\
0.6956 & 1.3794 & 0.0939 & 0.1557 \\
0.3686 & 0.0893 & 1.0429 & 0.0517 \\
0.3071 & 0.0012 & 0.0155 & 1.0062
\end{array}\right)
$$

and

$$
\int_{0}^{1} \mathcal{K}(s) s(1-s) d s>\left(\begin{array}{c}
31.1 \times 10^{-5} \\
288.9 \times 10^{-5} \\
137.5 \times 10^{-5} \\
27.4 \times 10^{-5}
\end{array}\right)
$$

It follows from Lemma 2.1, (2.25), and (2.26) that

$$
\frac{c_{1}}{2} \int_{0}^{1}\left(\kappa_{3}(s)+\kappa_{4}(s)\right) c_{2}(s) d s \geq \frac{c_{1}}{2} \int_{0}^{1}\left(\kappa_{3}(s)+\kappa_{4}(s)\right) s(1-s) d s>1
$$


since $\kappa_{3}(s)$ and $\kappa_{4}(s)$ are the third and the fourth components in $(I-[B])^{-1} \mathcal{K}(s)$ respectively, so (2.10) is valid. It can be seen that (2.11) is satisfied for $C_{0}$ large enough. Then BVP (2.22) has a positive solution by Theorem 2.1 .

Example 2.2 If $f\left(t, x_{1}, x_{2}, x_{3}\right)=\sqrt[3]{x_{1}}-\sqrt[3]{x_{3}}$, then BVP (2.22) has a positive solution.

Proof Take $a_{1}=1 / 2, b_{1}=0, c_{1}=1 / 3, C_{0}=2$ and $a_{2}=1043, b_{2}=0, c_{2}=1, r=1 / 33685$. Obviously, (2.23) for $i=1$ and (2.24) are satisfied, meanwhile conditions (2.19) and (2.20) are fulfilled. Then BVP (2.22) has a positive solution by Theorem 2.2.

\section{Inequalities of Green's function and positive solutions for (1.2)}

For BVP (1.2) we make the assumption:

$\left(C_{1}^{\prime}\right) g:[0,1] \times \mathbb{R}_{+}^{3} \rightarrow \mathbb{R}_{+}$is continuous.

Similar to Webb and Infante [16], BVP (1.2) can be converted to the integral equation in $C^{2}[0,1]$ :

$$
u(t)=(\widetilde{S} u)(t)=: \int_{0}^{1} \widetilde{k}_{S}(t, s) g\left(s, u(s), u^{\prime}(s), u^{\prime \prime}(s)\right) d s,
$$

where

$$
\widetilde{k}_{S}(t, s)=\left\langle(I-[A])^{-1} \widetilde{\mathcal{K}}(s), \delta(t)\right\rangle+\widetilde{k}_{0}(t, s)=\sum_{i=1}^{4} \widetilde{\kappa}_{i}(s) \delta_{i}(t)+\widetilde{k}_{0}(t, s),
$$

$\left\langle(I-[A])^{-1} \tilde{\mathcal{K}}(s), \delta(t)\right\rangle$ is the inner product in $\mathbb{R}^{4}$,

$$
\begin{array}{ll}
\widetilde{\mathcal{K}}_{i}(s):=\int_{0}^{1} \widetilde{k}_{0}(t, s) d A_{i}(t) & (i=1,2,3,4), \\
\delta_{1}(t)=1, & \delta_{2}(t)=t, \quad \delta_{3}(t)=\frac{1}{6} t^{2}(3-t), \quad \delta_{4}(t)=\frac{1}{6} t^{3},
\end{array}
$$

$\widetilde{\kappa}_{i}(s)$ is the $i$ th component of $(I-[A])^{-1} \widetilde{\mathcal{K}}(s)$,

$$
\widetilde{k}_{0}(t, s)= \begin{cases}\frac{1}{6} t^{3}(1-s), & 0 \leq t \leq s \leq 1, \\ \frac{1}{6} s\left(3 t^{2}-3 t s+s^{2}-t^{3}\right), & 0 \leq s \leq t \leq 1 .\end{cases}
$$

We put forward the following hypotheses:

$\left(C_{2}^{\prime}\right) A_{i}$ is of bounded variation and $\widetilde{\mathcal{K}}_{i}(s) \geq 0, \forall s \in[0,1](i=1,2,3,4)$;

$\left(C_{3}^{\prime}\right)$ The $4 \times 4$ matrix $[A]$ is positive whose $(i, j)$ th entry is $\alpha_{i}\left[\delta_{j}\right]$ and whose spectrum radius $r([A])<1$.

Lemma 3.1 If $\left(C_{2}^{\prime}\right)$ and $\left(C_{3}^{\prime}\right)$ hold, then $\widetilde{\kappa}_{i}(s) \geq 0(i=1,2,3,4)$ and, for $t, s \in[0,1]$,

$$
\widetilde{c}_{0}(t) \widetilde{\Phi}_{0}(s) \leq \widetilde{k}_{S}(t, s) \leq \widetilde{\Phi}_{0}(s),
$$

where

$$
\widetilde{\Phi}_{0}(s)=\sum_{i=1}^{4} \widetilde{\kappa}_{i}(s)+\frac{1}{6} s(1-s)(2-s), \quad \widetilde{c}_{0}(t)=\frac{1}{6} t^{3},
$$


and

$$
\widetilde{c}_{1}(t) \widetilde{\Phi}_{1}(s) \leq \frac{\partial \widetilde{k}_{S}(t, s)}{\partial t} \leq \widetilde{\Phi}_{1}(s), \quad \widetilde{c}_{2}(t) \widetilde{\Phi}_{2}(s) \leq \frac{\partial^{2} \widetilde{k}_{S}(t, s)}{\partial t^{2}} \leq \widetilde{\Phi}_{2}(s),
$$

where

$$
\begin{array}{ll}
\widetilde{\Phi}_{1}(s)=\sum_{i=2}^{4} \widetilde{\kappa}_{i}(s)+\frac{1}{2} s(1-s), & \widetilde{c}_{1}(t)=\frac{1}{2} t^{2}, \\
\widetilde{\Phi}_{2}(s)=\sum_{i=3}^{4} \widetilde{\kappa}_{i}(s)+s(1-s), & \widetilde{c}_{2}(t)=\min \{t, 1-t\} .
\end{array}
$$

Proof For $s \in[0,1], \widetilde{\kappa}_{i}(s) \geq 0(i=1,2,3,4)$ are due to [16, proof of Theorem 2.4] since both $(I-[A])^{-1}$ and $\widetilde{\mathcal{K}}(s)$ are nonnegative.

According to the following two inequalities:

$$
\begin{aligned}
& \frac{1}{6} t^{3} \sum_{i=1}^{4} \widetilde{\kappa}_{i}(s) \leq \sum_{i=1}^{4} \widetilde{\kappa}_{i}(s) \delta_{i}(t) \leq \sum_{i=1}^{4} \widetilde{\kappa}_{i}(s), \\
& \frac{1}{6} t^{3} \frac{1}{6} s(1-s)(2-s) \leq t^{3} \frac{1}{6} s(1-s)(2-s) \leq \widetilde{k}_{0}(t, s) \leq \frac{1}{6} s(1-s)(2-s),
\end{aligned}
$$

we have, for $t, s \in[0,1]$,

$$
\widetilde{c}_{0}(t) \widetilde{\Phi}_{0}(s) \leq \widetilde{k}_{S}(t, s)=\sum_{i=1}^{4} \widetilde{\kappa}_{i}(s) \delta_{i}(t)+\widetilde{k}_{0}(t, s) \leq \widetilde{\Phi}_{0}(s) .
$$

Moreover, the next two inequalities

$$
\begin{aligned}
& \frac{1}{2} t^{2} \sum_{i=2}^{4} \widetilde{\kappa}_{i}(s) \leq \sum_{i=1}^{4} \widetilde{\kappa}_{i}(s) \delta_{i}^{\prime}(t) \leq \sum_{i=2}^{4} \widetilde{\kappa}_{i}(s) \\
& \frac{1}{2} t^{2} \frac{1}{2} s(1-s) \leq t^{2} \frac{1}{2} s(1-s) \leq \frac{\partial \widetilde{k}_{0}(t, s)}{\partial t} \leq \frac{1}{2} s(1-s)
\end{aligned}
$$

imply, for $t, s \in[0,1]$,

$$
\widetilde{c}_{1}(t) \widetilde{\Phi}_{1}(s) \leq \frac{\partial \widetilde{k}_{S}(t, s)}{\partial t}=\sum_{i=1}^{4} \widetilde{\kappa}_{i}(s) \delta_{i}^{\prime}(t)+\frac{\partial \widetilde{k}_{0}(t, s)}{\partial t} \leq \widetilde{\Phi}_{1}(s) .
$$

Finally, from the two inequalities

$$
\begin{aligned}
& \min \{t, 1-t\} \sum_{i=3}^{4} \kappa_{i}(s) \leq \sum_{i=1}^{4} \widetilde{\kappa}_{i}(s) \delta_{i}^{\prime \prime}(t)=(1-t) \kappa_{3}(s)+t \kappa_{4}(s) \leq \sum_{i=3}^{4} \widetilde{\kappa}_{i}(s), \\
& \min \{t, 1-t\} s(1-s) \leq \frac{\partial^{2} \widetilde{k}_{0}(t, s)}{\partial t^{2}} \leq s(1-s)
\end{aligned}
$$

it follows that

$$
\widetilde{c}_{2}(t) \widetilde{\Phi}_{2}(s) \leq \frac{\partial^{2} \widetilde{k}_{S}(t, s)}{\partial t^{2}}=\sum_{i=1}^{4} \widetilde{\kappa}_{i}(s) \delta_{i}^{\prime \prime}(t)+\frac{\partial^{2} \widetilde{k}_{0}(t, s)}{\partial t^{2}} \leq \widetilde{\Phi}_{2}(s)
$$

for $t, s \in[0,1]$. 
Define the subsets in $C^{2}[0,1]$ as follows:

$$
\begin{aligned}
\widetilde{P}= & \left\{u \in C^{2}[0,1]: u(t) \geq 0, u^{\prime}(t) \geq 0, u^{\prime \prime}(t) \geq 0, \forall t \in[0,1]\right\}, \\
\widetilde{K}= & \left\{u \in \widetilde{P}: u(t) \geq c_{0}(t)\|u\|_{C}, u^{\prime}(t) \geq c_{1}(t)\left\|u^{\prime}\right\|_{C^{\prime}}\right. \\
& \left.u^{\prime \prime}(t) \geq c_{2}(t)\left\|u^{\prime \prime}\right\|_{C}, \forall t \in[0,1] ; \alpha_{i}[u] \geq 0(i=1,2,3,4)\right\} .
\end{aligned}
$$

Clearly both $\widetilde{P}$ and $\widetilde{K}$ are cones, and it is easy to check that $\widetilde{P}$ is a solid cone.

Now we define linear operators in $C^{2}[0,1]$ :

$$
\begin{aligned}
& \left(\widetilde{L}_{i} u\right)(t)=\int_{0}^{1} \widetilde{k}_{s}(t, s)\left(\widetilde{a}_{i} u(s)+\widetilde{b}_{i} u^{\prime}(s)+\widetilde{c}_{i} u^{\prime \prime}(s)\right) d s \quad(i=1,2), \\
& \left(\widetilde{L}_{3} u\right)(t)=\widetilde{a}_{1} \int_{0}^{1} \widetilde{k}_{S}(t, s) u(s) d s
\end{aligned}
$$

where $\widetilde{a}_{i}, \widetilde{b}_{i}, \widetilde{c}_{i}(i=1,2)$ are nonnegative constants.

Similar to [16], we have the following Lemma 3.2 by Lemma 3.1 .

Lemma 3.2 If $\left(C_{1}^{\prime}\right)-\left(C_{3}^{\prime}\right)$ hold, then $\widetilde{S}: \widetilde{P} \rightarrow \widetilde{K}$ and $\widetilde{L}_{i}: C^{2}[0,1] \rightarrow C^{2}[0,1]$ are completely continuous operators with $\widetilde{L}_{i}(\widetilde{P}) \subset \widetilde{K}(i=1,2,3)$, where $\widetilde{S}, \widetilde{P}, \widetilde{K}$ are defined separately in (3.1), (3.4), and (3.5).

Theorem 3.1 Under hypotheses $\left(C_{1}^{\prime}\right)-\left(C_{3}^{\prime}\right)$ suppose that

$\left(\widetilde{F}_{1}\right)$ there exist constants $\widetilde{a}_{2}, \widetilde{b}_{2}, \widetilde{c}_{2} \geq 0$, and $\widetilde{r}>0$ such that

$$
g\left(t, x_{1}, x_{2}, x_{3}\right) \leq \widetilde{a}_{2} x_{1}+\widetilde{b}_{2} x_{2}+\widetilde{c}_{2} x_{3}
$$

for all $\left(t, x_{1}, x_{2}, x_{3}\right) \in[0,1] \times[0, \widetilde{r}]^{3}$, moreover the spectral radius $r\left(\widetilde{L}_{2}\right)<1$, where $\widetilde{L}_{2}$ is defined by (3.6),

$\left[\left(\widetilde{F}_{2}\right)\right.$ there exist positive constants $\widetilde{a}_{1}, \widetilde{b}_{1}, \widetilde{c}_{1}, \widetilde{C}_{0}$ satisfying

$$
\begin{aligned}
& \min \left\{\frac{\widetilde{a}_{1}}{6} \int_{0}^{1} \widetilde{c}_{0}(s) \widetilde{\Phi}_{0}(s) d s, \frac{\widetilde{b}_{1}}{2} \int_{0}^{1} \widetilde{c}_{1}(s) \widetilde{\Phi}_{1}(s) d s,\right. \\
& \left.\frac{\widetilde{c}_{1}}{2} \int_{0}^{1}\left(\widetilde{\kappa}_{3}(s)+\widetilde{\kappa}_{4}(s)\right) c_{2}(s) d s\right\}>1
\end{aligned}
$$

such that

$$
g\left(t, x_{1}, x_{2}, x_{3}\right) \geq \widetilde{a}_{1} x_{1}+\widetilde{b}_{1} x_{2}+\widetilde{c}_{1} x_{3}-\widetilde{C}_{0}
$$

for all $\left(t, x_{1}, x_{2}, x_{3}\right) \in[0,1] \times \mathbb{R}_{+}^{3}$.

Then BVP (1.2) has one positive solution in $\widetilde{K}$.

Proof Let

$$
M=\max \left\{\frac{6 \widetilde{C}_{0} \int_{0}^{1} \widetilde{\Phi}_{0}(s) d s}{\widetilde{a}_{1} \int_{0}^{1} \widetilde{c}_{0}(s) \widetilde{\Phi}_{0}(s) d s-6}, \frac{2 \widetilde{C}_{0} \int_{0}^{1} \widetilde{\Phi}_{1}(s) d s}{\widetilde{b}_{1} \int_{0}^{1} \widetilde{c}_{1}(s) \widetilde{\Phi}_{1}(s) d s-2},\right.
$$




$$
\left.\frac{\widetilde{C}_{0} \int_{0}^{1}\left(\widetilde{\kappa}_{3}(s)+\widetilde{\kappa}_{4}(s)\right) d s}{c_{1} \int_{0}^{1}\left(\widetilde{\kappa}_{3}(s)+\widetilde{\kappa}_{4}(s)\right) \widetilde{c}_{2}(s) d s-2}\right\}
$$

and the rest is similar to the proof of Theorem 2.1 in which $\widetilde{c}_{0}(t)=\frac{1}{2} t^{3}$ for step (iv).

Theorem 3.2 Under hypotheses $\left(C_{1}^{\prime}\right)-\left(C_{3}^{\prime}\right)$, suppose that

$\left(\widetilde{F}_{3}\right)$ there exist constants $\widetilde{a}_{1}, \widetilde{b}_{1}, \widetilde{c}_{1}, \widetilde{C}_{0} \geq 0$ such that

$$
g\left(t, x_{1}, x_{2}, x_{3}\right) \leq \widetilde{a}_{1} x_{1}+\widetilde{b}_{1} x_{2}+\widetilde{c}_{1} x_{3}+\widetilde{C}_{0}
$$

for all $\left(t, x_{1}, x_{2}, x_{3}\right) \in[0,1] \times \mathbb{R}_{+}^{3}$, moreover the spectral radius $r\left(\widetilde{L}_{1}\right)<1$;

$\left(\widetilde{F}_{4}\right)$ there exist constants $\widetilde{a}_{2}, \widetilde{b}_{2}, \widetilde{c}_{2} \geq 0$, and $\widetilde{r}>0$ such that

$$
g\left(t, x_{1}, x_{2}, x_{3}\right) \geq \widetilde{a}_{2} x_{1}+\widetilde{b}_{2} x_{2}+\widetilde{c}_{2} x_{3}
$$

for all $\left(t, x_{1}, x_{2}, x_{3}\right) \in[0,1] \times[0, \widetilde{r}]^{3}$, moreover the spectral radius $r\left(\widetilde{L}_{2}\right) \geq 1$; where $\widetilde{L}_{i}: C^{2}[0,1] \rightarrow C^{2}[0,1](i=1,2)$ are defined by (3.6).

Then BVP (1.2) has one positive solution in $\widetilde{K}$.

Proof Denote the cone ordering induced by $\widetilde{P}, u \preceq v$ for $u, v \in X$ if and only if $v-u \in \widetilde{P}$ and equivalently $v \succeq u$. The rest is similar to the proof of Theorem 2.2.

As an example, we consider fourth-order boundary problem under mixed multi-point and integral boundary conditions with sign-changing coefficients:

$$
\begin{cases}-u^{(4)}(t)=f\left(t, u(t), u^{\prime}(t), u^{\prime \prime}(t)\right), & t \in[0,1], \\ u(0)=\frac{1}{2} u\left(\frac{1}{4}\right)-\frac{1}{60} u\left(\frac{3}{4}\right), & u^{\prime}(0)=\int_{0}^{1} u(t)\left(t-\frac{1}{8}\right) d t, \\ u^{\prime \prime}(0)=\frac{1}{2} u\left(\frac{1}{2}\right)-\frac{1}{16} u\left(\frac{3}{4}\right), & u^{\prime \prime}(1)=\frac{1}{2} u\left(\frac{1}{4}\right)-\frac{1}{40} u\left(\frac{1}{2}\right),\end{cases}
$$

thus $\alpha_{1}[u]=\frac{1}{2} u\left(\frac{1}{4}\right)-\frac{1}{60} u\left(\frac{3}{4}\right), \alpha_{2}[u]=\int_{0}^{1} u(t)\left(t-\frac{1}{8}\right) d t, \alpha_{3}[u]=\frac{1}{2} u\left(\frac{1}{2}\right)-\frac{1}{16} u\left(\frac{3}{4}\right)$, and $\alpha_{4}[u]=$ $\frac{1}{2} u\left(\frac{1}{4}\right)-\frac{1}{40} u\left(\frac{1}{2}\right)$. We estimate some coefficients, and Matlab is also used.

$$
\begin{aligned}
\widetilde{\mathcal{K}}_{1}(s) & =\int_{0}^{1} \widetilde{k}_{0}(t, s) d A_{1}(t)=\frac{1}{2} \widetilde{k}_{0}\left(\frac{1}{4}, s\right)-\frac{1}{60} \widetilde{k}_{0}\left(\frac{3}{4}, s\right) \\
& = \begin{cases}\frac{29}{360} s^{3}-\frac{9}{160} s^{2}+\frac{83}{7680} s, & 0 \leq s \leq \frac{1}{4}, \\
-\frac{1}{360} s^{3}+\frac{1}{160} s^{2}-\frac{37}{7680} s+\frac{1}{768}, & \frac{1}{4}<s \leq \frac{3}{4}, \\
-\frac{1}{7680} s+\frac{1}{7680}, & \frac{3}{4}<s \leq 1,\end{cases}
\end{aligned}
$$

and hence $0 \leq \widetilde{\mathcal{K}}_{1}(s)<0.0007$

$$
\begin{aligned}
\widetilde{\mathcal{K}}_{2}(s) & =\int_{0}^{1} \widetilde{k}_{0}(t, s)\left(t-\frac{1}{8}\right) d t \\
& =-\frac{1}{120} s^{5}+\frac{1}{192} s^{4}+\frac{1}{16} s^{3}-\frac{13}{96} s^{2}+\frac{73}{960} s \quad(0 \leq s \leq 1),
\end{aligned}
$$


and hence $0 \leq \widetilde{\mathcal{K}}_{2}(s)<0.0129$

$$
\begin{aligned}
\widetilde{\mathcal{K}}_{3}(s) & =\int_{0}^{1} \widetilde{k}_{0}(t, s) d A_{3}(t)=\frac{1}{2} \widetilde{k}_{0}\left(\frac{1}{2}, s\right)-\frac{1}{16} \widetilde{k}_{0}\left(\frac{3}{4}, s\right) \\
& = \begin{cases}\frac{7}{96} s^{3}-\frac{13}{128} s^{2}+\frac{239}{6144} s, & 0 \leq s \leq \frac{1}{2}, \\
-\frac{1}{96} s^{3}+\frac{3}{128} s^{2}-\frac{115}{6144} s+\frac{1}{96}, & \frac{1}{2}<s \leq \frac{3}{4}, \\
-\frac{37}{6144} s+\frac{37}{6144}, & \frac{3}{4}<s \leq 1,\end{cases}
\end{aligned}
$$

and hence $0 \leq \widetilde{\mathcal{K}}_{3}(s)<0.0046$

$$
\begin{aligned}
\widetilde{\mathcal{K}}_{4}(s) & =\int_{0}^{1} \widetilde{k}_{0}(t, s) d B_{4}(t)=\frac{1}{2} \widetilde{k}_{0}\left(\frac{1}{4}, s\right)-\frac{1}{40} \widetilde{k}_{0}\left(\frac{1}{2}, s\right) \\
& = \begin{cases}\frac{19}{240} s^{3}-\frac{9}{160} s+\frac{3}{256} s, & 0 \leq s \leq \frac{1}{4}, \\
-\frac{1}{240} s^{3}+\frac{1}{160} s^{2}-\frac{1}{256} s+\frac{1}{768}, & \frac{1}{4}<s \leq \frac{1}{2}, \\
-\frac{1}{1280} s+\frac{1}{1280}, & \frac{1}{2}<s \leq 1,\end{cases}
\end{aligned}
$$

and hence $0 \leq \widetilde{\mathcal{K}}_{4}(s)<0.0008$.

The $4 \times 4$ matrix

$$
[A]=\left(\begin{array}{llll}
\alpha_{1}\left[\delta_{1}\right] & \alpha_{1}\left[\delta_{2}\right] & \alpha_{1}\left[\delta_{3}\right] & \alpha_{1}\left[\delta_{4}\right] \\
\alpha_{2}\left[\delta_{1}\right] & \alpha_{2}\left[\delta_{2}\right] & \alpha_{2}\left[\delta_{3}\right] & \alpha_{2}\left[\delta_{4}\right] \\
\alpha_{3}\left[\delta_{1}\right] & \alpha_{3}\left[\delta_{2}\right] & \alpha_{3}\left[\delta_{3}\right] & \alpha_{3}\left[\delta_{4}\right] \\
\alpha_{4}\left[\delta_{1}\right] & \alpha_{4}\left[\delta_{2}\right] & \alpha_{4}\left[\delta_{3}\right] & \alpha_{4}\left[\delta_{4}\right]
\end{array}\right)=\left(\begin{array}{cccc}
\frac{29}{60} & \frac{9}{80} & \frac{83}{7680} & \frac{1}{7680} \\
\frac{3}{8} & \frac{13}{48} & \frac{73}{960} & \frac{9}{320} \\
\frac{7}{16} & \frac{13}{64} & \frac{239}{6144} & \frac{37}{6144} \\
\frac{19}{40} & \frac{9}{80} & \frac{3}{256} & \frac{1}{1280}
\end{array}\right)
$$

and its spectrum radius $r([A]) \approx 0.6444<1$. Those mean that $\left(C_{2}^{\prime}\right)$ and $\left(C_{3}^{\prime}\right)$ are satisfied.

Now we take stock of some constants in Theorem 3.1 and Theorem 3.2.

$$
(I-[A])^{-1}<\left(\begin{array}{llll}
2.2565 & 0.3651 & 0.0545 & 0.0110 \\
1.3460 & 1.6266 & 0.1445 & 0.0469 \\
1.3195 & 0.5123 & 1.0962 & 0.0213 \\
1.2398 & 0.3627 & 0.0551 & 1.0116
\end{array}\right)
$$

and

$$
(I-[A])^{-1} \widetilde{\mathcal{K}}(s)<\left(\begin{array}{c}
0.0065 \\
0.0226 \\
0.0126 \\
0.0066
\end{array}\right)
$$

thus $\widetilde{k}_{S}(t, s)<0.0065+0.0226 t+0.0126 \times \frac{1}{6} t^{2}(3-t)+0.0066 \times \frac{1}{6} t^{3}+\widetilde{k}_{0}(t, s)<0.0987$. So, for $u \in C^{2}[0,1]$ and $t \in[0,1]$,

$$
\begin{aligned}
\left.\mid \widetilde{L}_{i} u\right)(t) \mid & \leq 0.0987 \int_{0}^{1}\left(a_{i}|u(s)|+b_{i}\left|u^{\prime}(s)\right|+c_{i}\left|u^{\prime \prime}(s)\right|\right) d s \\
& \leq 0.0987\left(a_{i}+b_{i}+c_{i}\right)\|u\|_{C^{2}} \quad(i=1,2),
\end{aligned}
$$


here $\widetilde{L}_{i}(i=1,2)$ are defined in (3.6). Since all the terms are nonnegative in the first and second derivatives of $k_{S}(t, s)$ with respect to $t$, we also have that, for $u \in C^{2}[0,1]$ and $t \in$ $[0,1]$,

$$
\begin{aligned}
\left.\mid \widetilde{L}_{i} u\right)^{\prime}(t) \mid & \leq 0.1573 \int_{0}^{1}\left(a_{i}|u(s)|+b_{i}\left|u^{\prime}(s)\right|+c_{i}\left|u^{\prime \prime}(s)\right|\right) d s \\
& \leq 0.1573\left(a_{i}+b_{i}+c_{i}\right)\|u\|_{C^{2}} \quad(i=1,2), \\
\left|\left(\widetilde{L}_{i} u\right)^{\prime \prime}(t)\right| & \leq 0.2597 \int_{0}^{1}\left(a_{i}|u(s)|+b_{i}\left|u^{\prime}(s)\right|+c_{i}\left|u^{\prime \prime}(s)\right|\right) d s \\
& \leq 0.2597\left(a_{i}+b_{i}+c_{i}\right)\|u\|_{C^{2}} \quad(i=1,2) .
\end{aligned}
$$

Therefore the radius $r\left(\widetilde{L}_{i}\right) \leq\left\|L_{i}\right\| \leq 0.2597\left(a_{i}+b_{i}+c_{i}\right)<1$ if

$$
a_{i}+b_{i}+c_{i}<0.2597^{-1} \quad(i=1,2) .
$$

On the other hand, we have from Lemma 3.1 and Lemma 3.2 that, for $u \in \widetilde{K} \backslash\{0\}$ and $t \in[0,1]$,

$$
\begin{aligned}
\left(\widetilde{L}_{2} u\right)(t) & \geq \int_{0}^{1} \widetilde{k}_{S}(t, s) \widetilde{a}_{2} u(s) d s \geq \widetilde{a}_{2} \widetilde{c}_{0}(t) \int_{0}^{1} \widetilde{\Phi}_{0}(s) u(s) d s \\
& \geq \widetilde{a}_{2} \widetilde{c}_{0}(t) \int_{0}^{1} \widetilde{\Phi}_{0}(s) \widetilde{c}_{0}(s)\|u\|_{C} d s=\widetilde{a}_{2} \widetilde{c}_{0}(t)\|u\|_{C} \int_{0}^{1} \widetilde{c}_{0}(s) \widetilde{\Phi}_{0}(s) d s
\end{aligned}
$$

and

$$
\left\|\left(\widetilde{L}_{2} u\right)\right\|_{C}=\left(\widetilde{L}_{2} u\right)(1) \geq \frac{1}{6} \widetilde{a}_{2}\|u\|_{C} \int_{0}^{1} \widetilde{c}_{0}(s) \widetilde{\Phi}_{0}(s) d s
$$

hence

$$
\begin{aligned}
\left(\widetilde{L}_{2}^{2} u\right)(t) & \geq \widetilde{a}_{2} \int_{0}^{1} \widetilde{k}_{S}(t, s)\left(\widetilde{L}_{2} u\right)(s) d s \geq \widetilde{a}_{2} \widetilde{c}_{0}(t) \int_{0}^{1} \widetilde{\Phi}_{0}(s)\left(\widetilde{L}_{2} u\right)(s) d s \\
& \geq \widetilde{a}_{2} \widetilde{c}_{0}(t) \int_{0}^{1} \widetilde{\Phi}_{0}(s) \widetilde{c}_{0}(s)\left\|\left(\widetilde{L}_{2} u\right)\right\|_{C} d s \geq \frac{1}{6} \widetilde{a}_{2}^{2} \widetilde{c}_{0}(t)\|u\|_{C}\left(\int_{0}^{1} \widetilde{c}_{0}(s) \widetilde{\Phi}_{0}(s) d s\right)^{2}
\end{aligned}
$$

and

$$
\left\|\left(\widetilde{L}_{2}^{2} u\right)\right\|_{C}=\left(\widetilde{L}_{2}^{2} u\right)(1) \geq \frac{1}{36} \widetilde{a}_{2}^{2}\|u\|_{C}\left(\int_{0}^{1} \widetilde{c}_{0}(s) \widetilde{\Phi}_{0}(s) d s\right)^{2} .
$$

By induction,

$$
\left\|\left(\widetilde{L}_{2}^{n} u\right)\right\|_{C}=\left(\widetilde{L}_{2}^{n} u\right)(1) \geq\left(\frac{\widetilde{a}_{2}}{6}\right)^{n}\|u\|_{C}\left(\int_{0}^{1} \widetilde{c}_{0}(s) \widetilde{\Phi}_{0}(s) d s\right)^{n} .
$$

As a result, it follows that, for $u \in \widetilde{K} \backslash\{0\}$,

$$
\left\|\widetilde{L}_{2}^{n}\right\|\|u\|_{C^{2}} \geq\left\|\widetilde{L}_{2}^{n} u\right\|_{C^{2}} \geq\left\|\widetilde{L}_{2}^{n} u\right\|_{C} \geq\left(\frac{\widetilde{a}_{2}}{6}\right)^{n}\|u\|_{C}\left(\int_{0}^{1} \widetilde{c}_{0}(s) \widetilde{\Phi}_{0}(s) d s\right)^{n},
$$


and according to Gelfand's formula, the spectral radius

$$
\begin{aligned}
r\left(\widetilde{L}_{2}\right) & =\lim _{n \rightarrow \infty}\left\|\widetilde{L}_{2}^{n}\right\|^{1 / n} \\
& \geq \frac{\widetilde{a}_{2}}{6}\left(\int_{0}^{1} \widetilde{c}_{0}(s) \widetilde{\Phi}_{0}(s) d s\right) \lim _{n \rightarrow \infty}\left(\frac{\|u\|_{C}}{\|u\|_{C^{3}}}\right)^{1 / n}=\frac{\widetilde{a}_{2}}{6}\left(\int_{0}^{1} \widetilde{c}_{0}(s) \widetilde{\Phi}_{0}(s) d s\right),
\end{aligned}
$$

which implies that $r\left(\widetilde{L}_{2}\right) \geq 1$ when

$$
\widetilde{a}_{2} \geq 5040=\frac{6}{\int_{0}^{1} \frac{1}{6} s^{3} \times \frac{1}{6} s(1-s)(2-s) d s} \geq \frac{6}{\int_{0}^{1} \widetilde{c}_{0}(s) \widetilde{\Phi}_{0}(s) d s} .
$$

Example 3.1 If

$$
g\left(t, x_{1}, x_{2}, x_{3}\right)=\frac{\frac{3}{2} x_{1}^{5}+x_{2}^{5}+x_{3}^{5}}{1+x_{1}^{2}+x_{2}^{2}+x_{3}^{2}}
$$

then BVP (3.12) has a positive solution.

Proof Take $\widetilde{a}_{2}=3 / 2, \widetilde{b}_{2}=\widetilde{c}_{2}=1, r<1$, it is easy to check that (3.10) and (3.13) for $i=2$ are satisfied. Now take $\widetilde{a}_{1}=5040, \widetilde{b}_{1}=160, \widetilde{c}_{1}=990$, it is clear that

$$
\begin{aligned}
\frac{\widetilde{a}_{1}}{6} \int_{0}^{1} \widetilde{c}_{0}(s) \widetilde{\Phi}_{0}(s) d s & =\frac{\widetilde{a}_{1}}{36} \int_{0}^{1} s^{3} \widetilde{\Phi}_{0}(s) d s \\
& >\frac{\widetilde{a}_{1}}{36} \int_{0}^{1} \frac{1}{6} s^{4}(1-s)(2-s) d s \\
& =5040 \times \frac{1}{36} \int_{0}^{1} \frac{1}{6} s^{4}(1-s)(2-s) d s=1, \\
\frac{\widetilde{b}_{1}}{2} \int_{0}^{1} \widetilde{c}_{1}(s) \widetilde{\Phi}_{1}(s) d s & =\frac{\widetilde{b}_{1}}{4} \int_{0}^{1} s^{2} \widetilde{\Phi}_{1}(s) d s \\
& >\frac{\widetilde{b}_{1}}{4} \int_{0}^{1} \frac{1}{2} s^{3}(1-s) d s=160 \times \frac{1}{4} \int_{0}^{1} \frac{1}{2} s^{3}(1-s) d s=1 .
\end{aligned}
$$

We also have

$$
(I-[A])^{-1}>\left(\begin{array}{llll}
2.2563 & 0.3649 & 0.0543 & 0.0108 \\
1.3458 & 1.6264 & 0.1443 & 0.0467 \\
1.3193 & 0.5121 & 1.0960 & 0.0211 \\
1.2396 & 0.3625 & 0.0549 & 1.0114
\end{array}\right)
$$

and

$$
\int_{0}^{1} \tilde{\mathcal{K}}(s) s(1-s) d s>\left(\begin{array}{c}
3.2 \times 10^{-5} \\
150 \times 10^{-5} \\
48.6 \times 10^{-5} \\
6.7 \times 10^{-5}
\end{array}\right)
$$


It follows from Lemma 3.1, (3.15), and (3.16) that

$$
\frac{\widetilde{c}_{1}}{2} \int_{0}^{1}\left(\widetilde{\kappa}_{3}(s)+\widetilde{\kappa}_{4}(s)\right) c_{2}(s) d s \geq \frac{\widetilde{c}_{1}}{2} \int_{0}^{1}\left(\widetilde{\kappa}_{3}(s)+\widetilde{\kappa}_{4}(s)\right) s(1-s) d s>1
$$

since $\widetilde{\kappa}_{3}(s)$ and $\widetilde{\kappa}_{4}(s)$ are the third and the fourth components in $(I-[A])^{-1} \widetilde{\mathcal{K}}(s)$ respectively, so (3.8) is valid. It can be seen that (3.9) is satisfied for $\widetilde{C}_{0}$ large enough. Then BVP (3.12) has a positive solution by Theorem 3.1 .

Example 3.2 If $g\left(t, x_{1}, x_{2}, x_{3}\right)=\sqrt{x_{1}}+\sqrt{x_{4}}$, then BVP (3.12) has a positive solution.

Proof Take $\widetilde{a}_{1}=\widetilde{c}_{1}=1, \widetilde{b}_{1}=0, \widetilde{C}_{0}=1 / 2$ and $\widetilde{a}_{2}=5040, \widetilde{b}_{2}=0, \widetilde{c}_{2}=1, \widetilde{r}=3.9 \times 10^{-8}$. Obviously, (3.13) for $i=1$ and (3.14) are satisfied, meanwhile conditions (3.10) and (3.11) are fulfilled. Then BVP (3.12) has a positive solution by Theorem 3.2.

\section{Acknowledgements}

The authors express their sincere gratitude to the editors and anonymous referee for the careful reading of the original manuscript and thoughtful comments.

\section{Funding}

The project is supported by the Fundamental Research Funds for the Central Universities (N182410001) and the Training Program of Innovation and Entrepreneurship for Undergraduates of Northeastern University (201084).

\section{Availability of data and materials}

Not applicable.

\section{Competing interests}

The authors declare that they have no competing interests.

\section{Authors' contributions}

GZ provided the idea of this article, all authors completed the paper, read and approved the final manuscript.

\section{Publisher's Note}

Springer Nature remains neutral with regard to jurisdictional claims in published maps and institutional affiliations.

Received: 12 December 2019 Accepted: 7 April 2020 Published online: 21 April 2020

\section{References}

1. Alves, E., Ma, T.F., Pelicer, M.L.: Monotone positive solutions for a fourth order equation with nonlinear boundary conditions. Nonlinear Anal. 71, 3834-3841 (2009)

2. Bai, Z:: Positive solutions of some nonlocal fourth-order boundary value problem. Appl. Math. Comput. 215, 4191-4197 (2010)

3. Deimling, K.: Nonlinear Functional Analysis. Springer, Berlin (1985)

4. Guo, D., Lakshmikantham, V.: Nonlinear Problems in Abstract Cones. Academic Press, Boston (1988)

5. Guo, Y., Yang, F., Liang, Y.: Positive solutions for nonlocal fourth-order boundary value problems with all order derivatives. Bound. Value Probl. (2012). https://doi.org/10.1186/1687-2770-2012-29

6. Infante, G., Pietramala, P.: A cantilever equation with nonlinear boundary conditions. Electron. J. Qual. Theory Differ. Equ. (2009). https://doi.org/10.14232/ejatde.2009.4.15

7. Kaufmann, E.R., Kosmatov, N.: Elastic beam problem with higher order derivatives. Nonlinear Anal., Real World Appl. 8, 811-821 (2007)

8. Krasnosel'skii, M.A.: Positive Solutions of Operator Equations. Noordhoff, Groningen (1964)

9. Li, Y: Positive solutions of fourth-order boundary value problems with two parameters. J. Math. Anal. Appl. 281 477-484 (2003)

10. Li, Y: On the existence of positive solutions for the bending elastic beam equations. Appl. Math. Comput. 189, 821-827 (2007)

11. Li, Y.: Existence of positive solutions for the cantilever beam equations with fully nonlinear terms. Nonlinear Anal., Real World Appl. 27, 221-237 (2016)

12. Ma, R.: Existence of positive solutions of a fourth-order boundary value problem. Appl. Math. Comput. 168 1219-1231 (2005)

13. Ma, Y., Yin, C., Zhang, G.: Positive solutions of fourth-order problems with dependence on all derivatives in nonlinearity under Stieltjes integral boundary conditions. Bound. Value Probl. (2019). https://doi.org/10.1186/s13661-019-1155-7 
14. Minhós, F., Gyulov, T., Santos, A.I.: Lower and upper solutions for a fully nonlinear beam equation. Nonlinear Anal. 71, 281-292 (2009)

15. Wang, S., Chai, J., Zhang, G.: Positive solutions of beam equations under nonlocal boundary value conditions. Adv. Differ. Equ. (2019). https://doi.org/10.1186/s13662-019-2404-x

16. Webb, J.R.L., Infante, G.: Non-local boundary value problems of arbitrary order. J. Lond. Math. Soc. 79, 238-259 (2009)

17. Webb, J.R.L., Infante, G., Franco, D.: Positive solutions of nonlinear fourth-order boundary-value problems with local and non-local boundary conditions. Proc. R. Soc. Edinb. 138A, 427-446 (2008)

18. Yao, Q: Monotonically iterative method of nonlinear cantilever beam equations. Appl. Math. Comput. 205, 432-437 (2008)

19. Yao, Q.: Local existence of multiple positive solutions to a singular cantilever beam equation. J. Math. Anal. Appl. 363, 138-154 (2010)

Submit your manuscript to a SpringerOpen ${ }^{\circ}$ journal and benefit from:

- Convenient online submission

$\checkmark$ Rigorous peer review

Open access: articles freely available online

- High visibility within the field

- Retaining the copyright to your article

Submit your next manuscript at $\gg$ springeropen.com 